\title{
Agentes estressores e o enfrentamento de problemas em tenistas e mesatenistas universitários
}

\author{
Stressors and coping in tennis and table tennis college athletes
}

\author{
Guilherme Bagni, Kauan Galvão Morão, Renato Henrique Verzani, Afonso Antonio Machado
}

Universidade Estadual Paulista (UNESP), Rio Claro/SP, Brasil

\section{HISTÓRICO DO ARTIGO \\ Recebido: 25 março 2020 \\ Revisado: 16 julho 2020 \\ Aprovado: 18 julho 2020}

\section{PALAVRAS-CHAVE:}

Psicologia do Esporte; Estresse Psicológico; Adaptação Psicológica.

\section{KEYWORDS:}

Psychology; Sports; Stress; Psychological; Adaptation; Psychological.

\section{RESUMO}

INTRODUÇÃO: A avaliação e análise de agentes estressores e enfrentamento de problemas em atletas pode ter um papel importante na compreensão daquilo que é enfrentado por esses atletas no dia-a-dia, além de poder propiciar um aumento na performance esportiva. No caso específico dos atletas universitários, além das dificuldades do mundo esportivo em si, existem as questões que permeiam o ambiente universitário e, muitas vezes sem estrutura, os atletas tem que conciliar essas duas carreiras.

OBJETIVO: Analisar as estratégias de enfrentamento utilizadas por atletas universitários de esportes de raquete, bem como verificar as situações estressoras enfrentadas pelos mesmos.

MÉTODOS: Este estudo de caráter descritivo e quali-quantitivo, utilizou o survey como tipo de pesquisa. Foram analisados 9 atletas universitários de esportes de raquete. Como instrumento, foi utilizado o questionário Estratégias de Modos de Enfrentamento de Problemas. Para a análise dos dados, foi utilizada a estatística descritiva e a análise de conteúdo.

RESULTADOS: As estratégias de enfrentamento mais utilizadas pelos atletas foram as focadas no problema e busca por suporte social. Como agentes estressores notou-se a dificuldade na conciliação da carreira esportiva com a vida acadêmica.

CONCLUSÃO: Verificou-se que a maioria dos atletas utiliza estratégias de enfrentamento eficientes e encontra mais dificuldades em conciliar a dupla carreira (aluno-atleta) do que dificuldades esportivas em si. Assim, tornase necessário uma maior percepção por parte dos gestores do esporte universitário acerca deste tópico, sendo ainda necessário maiores estudos neste tópico, visando auxiliar os atletas e proporcionar um maior rendimento esportivo e acadêmico para os mesmos.

\section{ABSTRACT}

BACKGROUND: The evaluation and analysis of stressors and coping in athletes can play an important role in understanding what is faced by these athletes on a daily basis, in addition to providing an increase in sports performance. In college athletes, besides the difficulties of the sporting world itself, there are issues that permeate the university environment and, often without structure, athletes have to reconcile these two careers.

OBJECTIVE: Analyze the coping strategies used by university athletes of racket sports, as well as to verify the stressful situations faced by them.

METHODS: This study has a descriptive and quali-quantitative and qualifying approach and uses survey as a type of research. Nine college racquet sports athletes were analyzed. As a questionnaries, it was uses the Estratégias de Modos de Enfrentamento de Problemas. For data analysis, descriptive statistics and content analysis were used.

RESULTS: The coping strategies most used by athletes were those focused on the problem and the search for social support. As stressors, it was noted the difficulty in reconciling sports career with academic life.

CONCLUSION: It was found that most athletes uses effective coping strategies and have more difficulties to reconcile the dual career (student-athlete) than sports difficulties themselves. Thus, it is necessary to have a greater perception on the part of university sports managers about this topic, and further studies on this topic are necessary, aiming to help athletes and provide them with greater sports and academic performance. 


\section{INTRODUÇÃO}

A avaliação e análise de comportamentos e estados emocionais de atletas, equipes e técnicos por meio de instrumentos advindos da psicologia do esporte podem ter um papel importante na performance esportiva (CERIN, 2003; JONES, 2003). Aroni et al. (2019) relatam que esta temática vem sendo estudada há muitos anos e realizaram uma revisão sistemática, concluindo que ainda são necessários mais estudos, especialmente na análise da adaptação dos atletas no contexto esportivo. Frente a essa questão, estudar os problemas enfrentados pelos atletas universitários, bem como as estratégias de enfretamento que os mesmos utilizam, pode ser fundamental para propiciar um desenvolvimento do esporte universitário, bem como uma meIhora no desempenho esportivo.

De acordo com Barbosa (2017), um dos eixos esportivos considerado com grande potencial de crescimento e possibilidade de visibilidade no Brasil é o esporte universitário. Este eixo, cujo desenvolvimento vem abarcado por falta de investimento e pouco envolvimento dos poderes públicos, possui como principais participantes os atletas universitários. Além de todos os problemas enfrentados pelo esporte universitário brasileiro, esses atletas têm, muitas vezes, que conciliar o cotidiano acadêmico com a rotina de treinos, algo denominado dupla carreira (BORGGREFE; CACHAY, 2012; MELO; SOARES; ROCHA, 2014; VERZANI et al., 2018).

Para Lazarus e Folkman (1984), o enfrentamento de problemas (coping) pode ser definido por meio de duas vertentes teóricas: uma derivada da tradição da experimentação animal e a outra da psicologia do ego psicanalítico. A primeira os autores definem considerando o animal como dependente do seu sistema nervoso para tomar as medidas necessárias para sobreviverem. Na segunda, o enfrentamento é definido como pensamentos e atos que resolvem problemas e reduzem o nível de estresse. Seidl, Tróccoli e Zannon (2005) classificaram as estratégias de enfrentamento de problema em 4 dimensões, sendo estas: o enfrentamento focalizado no problema (onde o indivíduo foca no manejo ou modificação daquilo está que the causando estresse), o enfrentamento focalizado na emoção (está ligado com a regulação emocional do próprio indivíduo), a busca de suporte social (procura por apoio social para ajudar a lidar com a situação enfrentada) e a religiosidade e o pensamento fantasioso (utilização de fantasias, orações, dentre outros para o enfrentamento).

Verardi et al. (2011) analisaram as estratégias utilizadas por atletas de futebol, comparando os níveis amador e profissional, bem como as posições dos atletas. Como resultados os autores identificaram que a estratégia mais utilizada por ambos os grupos, em quase todas as posições, foi a estratégia focalizada no problema, com exceção dos laterais amadores que utilizam mais estratégias relacionadas ao pensamento fantasioso e a religiosidade.

Barbosa et al. (2013) analisaram as situações estressoras e as estratégias de enfrentamento utilizadas por atletas de futebol durante o período competitivo e identificaram como situações estressoras questões ligadas a problemas profissionais, ansiedade e auto insatisfação, sendo que a maioria desses atletas utiliza estratégias focadas no problema visando solucionar ou ao menos atenuar essas situações. Bagni et al. (2013) também estudaram atletas de futebol e identificaram que a maioria desses atletas utilizam estratégias de enfrentamento focado no problema.

Em estudo com 16 tenistas da primeira divisão do noroeste oeste da Inglaterra, Swettenham et al. (2018) verificaram que tenistas do sexo masculino verbalizaram um nível de estresse físico mais alto nos treinamentos, porém em relação ao desempenho o estresse foi maior na competição. Para as tenistas perceberam níveis mais altos de estresse externo e físico na condição de competição, porém em relação ao desempenho os níveis de estresse foram maiores nos treinamento. Em relação as estratégias de enfrentamento, a estratégia de enfrentamento focada no problema foi a mais utilizada pelos tenistas de ambos os sexos, tanto em treinamento quanto em competições.

Analisando 112 tenistas de alto nível do México e Estados Unidos, Puente-Díaz e Anshel (2005) verificaram como principais fontes de estresse comentários negativos de treinadores e familiares, além da trapaça do oponente. Carrasco et al. (2013) analisaram 155 jovens tenistas profissionais participantes de torneios na América do Sul e identificaram como principais estratégias de enfrentamento planejamento ativo, reestruturação cognitiva, calma emocional e busca de apoio social. Segundo os autores, tais estratégias propiciaram uma maior autonomia percebida, proporcionando maior bem-estar psicológico.

Kurimay, Pope-Rhodius e Kondric (2017) examinaram a relação entre ansiedade competitiva cognitiva e estratégias de enfrentamento em 102 mesatenistas nos Estados Unidos e verificaram existir alguma conexão entres a intensidade da ansiedade e as estratégias de enfrentamento, de modo que a alta ansiedade cognitiva resultou em alto uso de estratégias de evasão e focadas na emoção comparada com atletas com baixo nível de ansiedade cognitiva.

Em um estudo com praticantes de artes marciais, Souza Junior (2007) identificou que estes praticantes utilizam principalmente estratégias focadas no problema e a busca por suporte social. O autor também identificou um aumento da busca por suporte social conforme aumentava o tempo de prática da modalidade.

Ferreira (2014) analisou 187 estudantes universitário em Portugal, como resultados, foi notada uma forte correlação o estresse acadêmico e ansiedade nas provas, pressões avaliativas e pressões parentais correlacionam-se fortemente com a preocupação e tensão. Como estratégias de enfretamento foi verificado que mulheres utilizaram mais o suporte social e emocional que homens, enquanto estudantes do sexo masculino apresentaram maior utilização de outras substâncias, como medicamentos e álcool, em comparação com estudantes do sexo feminino. Também foi notado que a religião não apresentou resultado significativo na relação entre estratégias de enfrentamento e satisfação acadêmica.

Bagni et al. (2014) analisaram 93 atletas universitário e identificou como principais estratégias de enfretamento utilizadas a focada no problema e a busca por suporte social. Em relação aos agentes estressores o autor identificou 11 fatores, sendo a dificuldade de conciliar os treinamentos com a rotina acadêmica, a falta de apoio da universidade, o excesso de avaliações acadêmicas e o baixo desempenho acadêmico os que apresentaram maior percentual. 
Dentro da classificação dos esportes de raquete, a análise de atletas de tênis de campo e tênis de mesa se deve ao fatos destas modalidades serem pertencentes a campeonatos universitários. Estudar tais modalidades possibilita verificar as situações estressoras bem como as estratégias de enfrentamento utilizadas em modalidade onde os atletas permanecem no espaço da competição sem ter contato a todo o momento com o(a) técnico (a). Considerando tal fato, ressalta-se que os atletas devem, portanto, ter extrema consciência acerca deste processo estressor e de como podem enfrentar o estresse (SAMULSKI, 2011).

Diante disso, o objetivo foi analisar as estratégias de enfrentamento utilizadas por atletas universitários de esportes de raquete, bem como verificar as situações estressoras enfrentadas pelos mesmos.

\section{MÉTODOS}

Este estudo de caráter descritivo e quali-quantitivo, utilizou o survey como tipo de pesquisa, mais especificamente o questionário (THOMAS; NELSON; SILVERMAN, 2012). O projeto foi aprovado pelo comitê de ética do Instituto de Biociências da Universidade Estadual Paulista "Júlio de Mesquita Filho", sob o Parecer $n^{\circ}$. 2.119.052.

A amostra do estudo foi constituída por nove atletas universitários de esportes de raquete, sendo sete de tênis de mesa (seis do sexo masculino e um do sexo feminino) e dois de tênis de campo (um do sexo masculino e um do sexo feminino). Todos os atletas pertenciam a universidades públicas, sete desses atletas vinculados a universidades estaduais e dois a universidades federais. A média de idade dos atletas era de $21,56 \pm 1,81$ anos e tempo de prática médio de $5,11 \pm 4,46$ anos. Nenhum dos atletas recebia bolsa para a prática esportiva, porém todos representavam a universidade em competições.

Como instrumento, foi utilizado o questionário Estratégias de Modos de Enfrentamento de Problemas - EMEP (GIMENES; QUEIROZ, 1997 apud SEIDL; TRÓCCOLI; ZANNON, 2005, p. 190). Este questionário é composto por 47 perguntas, sendo 45 fechadas e 2 abertas. As questões fechadas são divididas em quatro dimensões: enfrentamento focalizado no problema, enfrentamento focalizado na emoção, busca de suporte social e pensamento fantasioso e religiosidade. As respostas são dadas em escala do tipo Likert de 1 - "eu nunca faço isso" - até 5 - "eu faço isso sempre". As perguntas abertas visavam descrever uma situação estressora que o atleta vinha enfrentando naquele momento e qual a estratégia de enfrentamento que ele vinha utilizando no momento, sendo que cada atleta poderia relatar mais de uma situação, bem como mais de uma estratégia.

Para a análise dos dados, foi utilizado o programa Excel. Após os dados serem tabulados utilizou-se a estatística descritiva para obtenção das médias e desvio-padrão das questões fechadas e dos dados sócio demográficos. As questões fechadas foram agrupadas nas quatro dimensões do questionário. Para as questões abertas foi utilizada a análise de conteúdo (BARDIN, 2011), onde as respostas foram analisadas e categorizadas.

\section{RESULTADOS}

Os resultados foram divididos em três Tabelas. A Tabela 1 contêm a média e o desvio padrão das questões fechadas, sendo que a estratégia de enfrentamento focada no problema foi a com maior média $(3,68 \pm 1,04)$, seguida da busca por suporte social $(3,58 \pm 1,02)$.

Tabela 1. Média e desvio padrão dos resultados das questões fechadas.

\begin{tabular}{lc}
\hline Estratégias de enfrentamento & Média e Desvio Padrão \\
\hline Focada no problema & $3,68 \pm 1,04$ \\
Focada na emoção & $2,34 \pm 1,02$ \\
Busca por suporte social & $3,58 \pm 0,94$ \\
Religiosidade e pensamento fantasioso & $2,13 \pm 1,06$
\end{tabular}

$\mathrm{Na}$ Tabela 2 são apresentados os agentes estressores descritos pelos atletas, sendo estes: problemas acadêmicos (50\%), problemas de desempenho esportivo (20\%), problemas de conciliar a carreira esportiva com demais atividades (20\%) e problemas físicos (10\%).

Tabela 2. Frequência absoluta e relativa dos agentes estressores enfrentados.

\begin{tabular}{ll}
\hline Agentes Estressores & $\mathbf{f ( \% )}$ \\
\hline Problemas Acadêmicos & $5(50)$ \\
Problemas de desempenho esportivo & $2(20)$ \\
Problemas físicos & $1(10)$ \\
$\begin{array}{l}\text { Problemas de conciliar a carreira esportiva com } \\
\text { demais atividades }\end{array}$ & $2(20)$ \\
\hline
\end{tabular}

A Tabela 3 apresenta as estratégias de enfrentamento de problemas descritas pelos atletas na segunda questão aberta, sendo a focada no problema a que apresentou os maiores valores (67\%), indo de encontro ao resultado apresentado na Tabela 1.

Tabela 3. Frequência absoluta e relativa das estratégias de enfrentamento de problemas.

\begin{tabular}{lc}
\hline Estratégias & $\mathbf{f ( \% )}$ \\
\hline Focada no problema & $8(67)$ \\
Focada na emoção & $2(16,5)$ \\
Focadas na busca de suporte social & $2(16,5)$ \\
\hline
\end{tabular}

A estratégia de enfrentamento focada no problema foi a que apresentou maior destaque nas Tabelas 1 e 3, indo de encontro ao estudo de Souza Junior (2007), Verardi et al. (2011), Barbosa et al. (2013), Bagni et al. (2013), Bagni et al. (2014) e Swettenham et al. (2018). Para Folkman (1991), essa estratégia pode ser uma das mais eficientes quando o indivíduo tem a situação sob controle. Gould, Eklund e Jackson (1993) e Nieuwenhuys et al. (2011) ressaltam que diferentes estratégias de enfrentamento podem ser efetivas, dependendo da situação e da automação das mesmas.

A busca por suporte social, que apresentou destaque na Tabela 1 e também foi identificada na Tabela 3 é considerada por diversos autores como Holt, Hoar e Frase (2005), Rees, Hardy e Freeman (2007), Kristiansen e Roberts (2010), Carrasco et al. (2013) e Bagni et al. (2014), como um fator eficiente no enfrentamento de problemas. Nesse caso em especial, os universitários acabam recebendo apoio de outros alunos e atletas, 
pois muitos acabam estudando em Universidades fora do local onde suas famílias residem e, além disso, diversos campeonatos acabam ocorrendo em outras cidades, de modo a terem uma rotina de viagens similar à de atletas de esporte "não-universitário" composto por viagens, ambientes de alojamento, estar em um local desconhecido, dentre outros, como apontado por Barbosa (2017). Brandão e Machado (2008) relatam a importância do profissional de psicologia do esporte nas viagens, sendo que este deve saber lidar com eventos que possam ocorrer nas viagens, especialmente aqueles ligados a situações estressoras. Os autores exemplificam tal situação com o simples auxílio na checagem da documentação, tanto para a viagem, quanto para a competição.

Brandão e Machado (2008) ainda apontam para o fato dos atletas poderem ser "contaminados" por questões familiares e midiáticas externas, algo que segundo Morão et al. (2016) e Bagni et al. (2018), se torna muito mais habitual atualmente por meio das mídias sociais. Assim, segundo Morão et al. (2016), o ambiente de reclusão esportiva (ambiente em que os atletas ficam alojados antes de uma partida ou competição) acaba não tendo o mesmo efeito e pode até ter efeitos negativos na visão de atletas. Entretanto, para Bagni et al. (2017), isso ainda não é um consenso entre atletas e comissão técnica. Por outro lado, alguns estudos como de Morão et al. (2016), Morão et al. (2017) e Bagni (2018) identificaram que os aparelhos eletrônicos e as redes sociais virtuais facilitaram o contato com amigos e familiares, minimizando a falta que alguns atletas sentiam dessas pessoas e reduzindo esse possível fator estressor.

O auxílio na questão da ambientação, tais como aspectos culturais, geográficos, ambientais, dentre outros, é apontada por Brandão e Machado (2008) como um fator relevante a ser analisado. Bagni et al. (2018) identificaram que este risco vem sendo minimizado pelas novas mídias, já que atletas relaram que os aparelhos eletrônicos facilitam a adaptação em cidades diferentes, pois auxilia no conhecimento prévio sobre a cidade, dentre outras questões destacadas.

Rees, Hardy e Freeman (2007), em uma pesquisa com golfistas, afirmaram que independentemente do nível de estresse, o suporte social pode ter ajudado diretamente o desempenho. Holt, Hoar e Frase (2005) realizaram uma revisão acerca do coping e afirmaram a importância de se analisar as etapas de desenvolvimento e o contexto em geral para uma maior efetividade dessas estratégias, sendo o suporte social o mais evidenciado em idades menores. Laborde et al. (2012) também ressaltaram que o contexto da situação pode influenciar a estratégia de coping utilizada.

Nota-se ainda que dos agentes estressores descritos na Tabela 2, muitos estão relacionados com questões da Universidade, corroborando com Ferreira (2014) acerca do estresse da vida acadêmica. Assim, aparentemente, os atletas enfrentam uma maior dificuldade em conciliar a carreira esportiva com a vida universitária do que propriamente encontram dificuldades no esporte, como mencionado por Bagni et al. (2014). Tal fato corrobora com as afirmações de Barbosa (2017) de que não ocorre um planejamento para que possa existir o aluno-atleta, mesmo que este represente a universidade em campeonatos, parece ser dever apenas do atleta arcar com as faltas, trabalhos, dentre outros que ele acaba perdendo devido à campeonatos e treinamentos. Estes fatores tornam difícil a chamada dupla carreira descrita por Borggrefe e Cachay (2012), Melo, Soares e Rocha (2014) e Verzani et al. (2018).

\section{CONCLUSÃO}

Verificou-se que a maioria dos atletas utiliza estratégias de enfrentamento focadas no problema e busca por suporte social, encontrando dificuldades em conciliar a dupla carreira (aluno-atleta). Desta forma, torna-se necessário uma maior percepção por parte dos gestores do esporte universitário, bem como de Instituições de Ensino Superior para que trabalhem junto na melhoria dessa vertente esportiva. Os agentes estressores aqui descritos podem influenciar negativamente o desempenho de um atleta que está preocupado com a prova do dia seguinte, mesmo sendo que naquele final de semana ele esteja disputando um campeonato importante.

Ainda são necessários mais estudos acerca do esporte universitário, especialmente a respeito da dupla carreira esportiva e de agentes estressores neste meio. Uma sugestão de estudo futuro seria verificar os agentes estressores, as estratégias de enfrentamento e o desempenho dos atletas, se possível a longo prazo. Cabe também a ressalva da importância do trabalho de um profissional de psicologia do esporte nessas modalidades para auxiliar os atletas nesta dupla carreira, bem como outras questões pertinentes.

\section{REFERÊNCIAS}

ARONI, A.; BATISTA, M.; REBUSTINI, F.; MACHADO, A.; GOMES, R. O estresse e o coping no esporte. In: PETRICA, J.; MESQUITA, H.; BATISTA, M.; MENDES, P. (Eds.). Psicologia do desporto e do exercício: abordagens académicas de investigação. Idanha a Nova: Edição Câmara Municipal de Idanha a Nova, 2019. p. $35-43$.

BAGNI, G.; BARBOSA, C. G.; MORÃO, K. G.; MACHADO, A. A. O coping no esporte universitário. In: Congresso de Iniciação Científica da USP - Unicamp - Unesp, 6., 2014, Rio Claro. Anais... Campinas: Unicamp, 2014. p. 1-2.

BAGNI, G.; MACHADO, A. A.; BARBOSA, C. G.; VERZANI, R. H.; MORÃO, K. G. Estratégias de enfrentamento de problemas em jovens atletas: um estudo através do EMEP. Coleção Pesquisa em Educação Física, Várzea Paulista, v. 12 , n. 2, p. 63-70, 2013.

BAGNI, G.; MORÃO, K. G.; VERZANI, R. H.; ARONI, A. A.; MACHADO, A. A. Influências das redes sociais no esporte. In: Congresso Internacional de Psicologia do Esporte, Desenvovimento Humano e Tecnologias, v. 3, 2017. Rio Claro. Anais... Rio Claro: UNESP, 2017. p. 72-72.

BAGNI, G.; MORÃO, K. G.; VERZANI, R. H.; MACHADO, A. A. Jovens atletas e as mídias sociais: percepções e alterações no contexto esportivo. Revista Brasileira de Futsal e Futebol, São Paulo, v. 10, n. 40, supl. 1, p. 531-7, 2018.

BARBOSA, C. G. A gestão pública do esporte universitário brasileiro: a bola não deve entrar por acaso. 2017. 95f. Tese (Doutorado em Desenvolvimento Humano e Tecnologias) - Universidade Estadual Paulista, Rio Claro, 2017.

BARBOSA, C G · MOURA, R · BAGNI, G · VERZANI, R · GONÇALVES, L. F · MORÃO, G.; MACHADO, A. A. Análise de estratégias contextuas de enfrentamento de problema em jogadores juniores de futebol de campo. Coleção Pesquisa em Educação Física, Várzea Paulista, v. 12, n. 1, p. 31-8, 2013.

BARDIN, L. Análise de conteúdo. São Paulo: Edições 70, 2011.

BORGGREFE, C.; CACHAY, K. "Dual careers": the structural coupling of elite sport and school exemplified by the german verbundsysteme. European Journal for Sport and Society, Abingdon, v. 9, n. 1-2, p. 57-80, 2012.

BRANDÃO, M. R. F.; MACHADO, A. A. Viajando com a equipe: o papel do psicólogo do esporte. Motriz, Rio Claro, v. 14, n. 4, p. 513-518, 2008.

CARRASCO, A. E. R.; CAMPBELL, R. Z.; LÓPEZ, A. L.; POBLETE, I. L.; GARCÍA-MAS, A. Autonomy, coping strategies and psychological well-being in young professional tennis players. Spanish Journal of Psychology, Madrid, v. 16, n. e75, p. 1-11, 2013.

CERIN, E. Anxiety versus fundamental emotions as predictors of perceived functionality of pre-competitive emotional states, threat, and challenge in 
individual sports. Journal of Applied Sport Psychology, Philadelphia, v. 15, n. 3, p. 223-38, 2003

FERREIRA, E. S. D. Ansiedade aos exames em estudantes universitários: relação com stresse académico, estratégias de coping e satisfação académica. 2014. 93f. Dissertação (Mestrado em Psicologia, Aconselhamento e Psicoterapia) - Universidade Lusófona de Humanidades e Tecnologias, Lisboa, 2014.

FOLKMAN, S. Coping across the life span: theoretical issues. In: CUMMINGS, E. M.; GREENE, A. L.; KARRAKER, K. H. (Eds.). Life-span developmental psychology: perspectives on stress and coping. Hillsdale: Erlbaum, 1991. p. 3-19.

GOULD, D.; EKLUND, R. C.; JACKSON, S. A. Coping strategies used by US olympic wrestlers. Research Quarterly for Exercise and Sport, Abingdon, v. 64, n. 1, p. 83-93, 1993

HOLT, N. L.; HOAR, S.; FRASER, S. N. How does coping change with development? A review of childhood and adolescence sport coping research. European Journal of Sport Science, Abingdon, v. 5, n. 1, p. 25-39, 2005.

JONES, M. V. Controlling emotions in sport. The Sport Psychologist, Champaign, v. 17, n. 4, p. 471-86, 2003.

KRISTIANSEN, E.; ROBERTS, G. C. Young elite athletes and social support: coping with competitive and organizational stress in "Olympic" competition. Scandinavian Journal of Medicine \& Science in Sports, Chichester, v. 20, n. 4, p. 686-95, 2010.

KURIMAY, D.; POPE-RHODIUS, A.; KONDRIC, M. The Relationship between stress and coping in table tennis. Journal of Human Kinetics, Champaign, v. 55, n. 1 , p. $75-81,2017$

LABORDE, S.; YOU, M.; DOSSEVILLE, F.; SALINAS, A. Culture, individual differences, and situation: Influence on coping in French and Chinese table tennis players. European Journal for Sport and Society, Abingdon, v. 12, n. 3, p. 255-261, 2012.

LAZARUS R.S.; FOLKMAN, S. Stress, appraisal, and coping. New York: Springer Publishing Company, Inc, 1984

MELO, L. B. S.; SOARES, A. J. G.; ROCHA, H. P. A. Perfil educacional de atletas em formação no futebol no estado do Rio de Janeiro. Revista Brasileira de Educação Física e Esporte, São Paulo, v. 28, n. 4, p. 617-28, 2014.

MORÃO, K. G.; BAGNI, G.; VERZANI, R. H.; BARBOSA, C. G.; MACHADO, A. A. A reclusão/concentração esportiva para atletas de categorias de base do futebol brasileiro. Revista Portuguesa de Ciências do Desporto, Porto, v. 16, n. 3, p. 78-89, 2016.

MORÃO, K. G. SENA, A. B. D.; BAGNI, G.; VERZANI, R. H.; MACHADO, A. A Novas mídias digitais, redes sociais virtuais e suas implicações no esporte. Coleção Pesquisa em Educação Física, Várzea Paulista, v. 16, n. 1, p. 89-96, 2017.

NIEUWENHUYS, A.; VOS, L.; PIJPSTRA, S.; BAKKER, F. C. Meta experiences and coping effectiveness in sport. Psychology of Sport and Exercise, Amsterdam, v. 12, n. 2, p. 135-43, 2011

PUENTE-DÍAZ, R.; ANSHEL, M. H. Sources of acute stress, cognitive appraisal, and coping strategies among highly skilled Mexican and U.S. competitive tennis players. The Journal of Social Psychology, Washington, v. 145, n. 4 p. 429-46, 2005.

REES, T.; HARDY, L.; FREEMAN, P. Stressors, social support, and effects upon performance in golf. Journal of Sports Sciences, Abingdon, v. 25, n. 1, p. 33-42, 2007

SAMULSKI, D. Treinamento mental no tênis: como desenvolver as habilidade mentais. Barueri: Manole, 2011.

SEIDL, E. M. F. TRÓCCOL, B. T.; ZANNON C. M. L. C. Pessoas vivendo com HIV/AIDS: enfrentamento, suporte social e qualidade de vida. Psicologia: Reflexão e Crítica, Porto Alegre, v. 18, n. 2, p. 188-95, 2005

SOUZA JUNIOR, O. M. de. Estratégias de enfrentamento utilizadas por praticantes de artes marciais. 2007. 84f. Dissertação (Mestrado em Psicologia da Saúde) - Universidade Metodista de São Paulo, São Bernardo do Campo, 2007.

SWETTENHAMA, L.; EUBANK, M. WON, D.; WHITEHEAD, A. E. Investigating stress and coping during practice and competition in tennis using think aloud. International Journal of Sport and Exercise Psychology, New York, v. 18 , n. 2, p. 1-21, 2018

THOMAS, J. R.; NELSON, J. K.; SILVERMAN, S. J. Métodos de pesquisa em atividade física. 6. ed. Porto Alegre: Artmed, 2012.

VERARDI C. E. L.; NEIVA, C. M.; PESSOA FILHO, D. M.; NAGAMINE, K. K.; MIYAZAKI, M. C. D. O. S. Estratégias de enfrentamento em jogadores de futebol. Revista Brasileira de Ciência e Movimento, Brasília, v. 19, n. 4, p.
60-7. 2011.

VERZANI, R H · MORÃO, K G · BAGNI, G · MACHADO, A. A. SERAPIÃO, A B. S. Desafios da dupla carreira na formação de futebolistas: olhar sobre escolaridade. Arquivos de Ciências do Esporte, Uberaba, v. 6, n. 3, p. 110113, 2018.

\section{AGRADECIMENTOS}

Os autores agradecem ao LEPESPE (Laboratório de Estudos e Pesquisas em Psicologia do Esporte) por auxiliar na execução desta pesquisa.

\section{CONFLITO DE INTERESSE}

Os autores do estudo declaram não haver conflito de interesses.

\section{FINANCIAMENTO}

Este estudo não teve apoio financeiro.

\section{ORCID E E-MAIL DOS AUTORES}

Guilherme Bagni (Autor Correspondente)

ORCID: 0000-0001-6246-2663.

E-mail: guilhermebagni@uol.com.br

Kauan Galvão Morão

ORCID: 0000-0002-3133-2994.

E-mail: kauangm@hotmail.com

Renato Henrique Verzani

ORCID: 0000-0002-4416-7668.

E-mail: renato_verzani@hotmail.com

Afonso Antonio Machado

ORCID: 0000-0002-5669-5425

E-mail: afonsoa@gmail.com 